Voix et Images

voixetimages

\title{
Bibliographie de François Charron
}

Volume 16, numéro 3 (48), printemps 1991

François Charron

URI : https://id.erudit.org/iderudit/200922ar

DOI : https://doi.org/10.7202/200922ar

Aller au sommaire du numéro

Éditeur(s)

Université du Québec à Montréal

ISSN

0318-9201 (imprimé)

1705-933X (numérique)

Découvrir la revue

Citer ce document

(1991). Bibliographie de François Charron. Voix et Images, 16(3), 469-480.

https://doi.org/10.7202/200922ar

Ce document est protégé par la loi sur le droit d'auteur. L'utilisation des services d'Érudit (y compris la reproduction) est assujettie à sa politique d'utilisation que vous pouvez consulter en ligne.

https://apropos.erudit.org/fr/usagers/politique-dutilisation/
Cet article est diffusé et préservé par Érudit.

Érudit est un consortium interuniversitaire sans but lucratif composé de l’Université de Montréal, l'Université Laval et l'Université du Québec à Montréal. Il a pour mission la promotion et la valorisation de la recherche. https://www.erudit.org/fr/ 


\section{Bibliographie de François Charron}

\section{GUVRES}

\section{I.1 Volumes}

18. Assauts, Libos (Lot-et-Garonne), [s.é.], 1972, [n.p.]. (Génération, no 7). Reproduit dans $A u$ "sujet » de la poésie, p. 9-28.

Au «sujet " de la poésie, Montréal, l'Hexagone, 1972, 54 p.

Projet d'écriture pour l'été '76, Montréal, les Herbes rouges, 1973, [n.p.] (n 12).

La Traversée / Le Regard, Montréal, les Herbes rouges, 1973, [n.p.] ( $\mathrm{n}^{\circ} 13$ )

[Sous le pseudonyme d'André Lamarre].

Littérature / Obscénités, Montréal, les Éditions Danielle Laliberté, 1973, 85 p.

Interventions politiques, Montréal, l'Aurore, 1974, 69 p. (Lecture en vélocipède, $\mathrm{n}^{0} 10$ ).

Persister et se maintenir dans les vertiges de la terre qui demeurent sans fin, Montréal, l'Aurore, 1974, 60 p. (Lecture en vélocipède, $n^{\circ} 5$ ).

Pirouette par hasard poésie, présentation de Gaétan Brulotte, Montréal, l'Aurore, 1975, 128 p. (Lecture en vélocipède, $n^{0} 18$ ).

Enthousiasme, Montréal, les Herbes rouges, 1976,52 p. Ill. (n ${ }^{08} 42-43$ ).

Du commencement à la fin, illustrations de Carole Massé, Montréal, les Herbes rouges, 1977,60 p. ( $n^{08} 47-48$ ).

Propagande illustrations de Serge Bruneau, Montréal, les Herbes rouges, 1977, [n. p.] ( n $\left.^{\circ} 55\right)$.

Blessures, avec des dessins de l'auteur, Montréal, les Herbes rouges, 1978, 67 p. Пl. ( $\mathrm{n}^{\circ 8} 67-68$ ); réédité en 1985, 69 p. Prix Émile-Nelligan 1979.

Feu, Montréal, les Herbes rouges, 1978, 33 p. Ml. (nº 64).

Le Temps échappé des yeux. Notes sur l'expérience de la peinture, Montréal, les Herbes rouges, 1979,58 p. Ill. ( $\left.n^{\infty 8} 75-76\right)$.

Peinture automatiste précédé de Qui parle dans la théorie?, Montréal, les Herbes rouges, 1979, 136 p. Ml. (Lecture en vélocipède).

1980, Montréal, les Herbes rouges, 1981, 81 p. Ill. (Lecture en vélocipède, $\mathrm{n}^{\circ} 26$ ). Mystère, Montréal, les Herbes rouges, 1981,35 p. ( $n^{\circ} 95$ ).

La Passion d'autonomie. Littérature et nationalisme, Montréal, lès Herbes rouges, 1982,69 p. ( $\left.{ }^{06} 99-100\right)$.

Toute parole m'éblouira, Montréal, les Herbes rouges, 1982, 76 p. (n ${ }^{\text {os }} 104-105$ ). 
D'où viennent les tableaux? Montréal, les Herbes rouges, 1983, 96 p. (n ${ }^{\circ 8} 110$ 112).

Je suis ce que je suis. Journal, Montréal, les Herbes rouges, 1983, 103 p. (Lecture en vélocipède).

François, Montréal, les Herbes rouges, 1984, 107 p.

La vie n'a pas de sens, Montréal, les Herbes rouges, 1985, 58 p. ( $n^{\circ} 134$ ).

La Chambre des miracles, Montréal, les Herbes rouges, '1986, 71 p. (n ${ }^{\text {os }} 151$ 152).

Le Fait de vivre ou d'avoir vécu, Montréal, les Herbes rouges, 1986, 144 p. (Poésie).

La Fragilité des choses, Montréal, les Herbes rouges, 1987, 50 p. (n 159).

Le Monde comme obstacle, Montréal, les Herbes rouges, 1988, 211 p. (Poésie).

La beauté pourrit sans douleur suivi de la Très Précieuse Qualité du vide, Montréal, les Herbes rouges, 1989, 189 p. (Poésie).

La beauté des visages ne pèse pas sur la terre, Trois-Rivières, Écrits des Forges, 1990, 137 p. (Radar, n 54). Grand prix de poésie 1990 de la Fondation les Forges.

\section{I.2. Poèmes parus dans des périodiques}

«Hyp(r)othèse ", Ether, 3, novembre 1970, p. 24-26.

«Un peu de Nouvelle-France du thème text-», la Barre du jour, 27, février 1971, p. 7-11.

*Déjà vu ", la Barre du jour, 28, mai 1971, p. 11-24.

«Big Hits ", la Barre du jour, 29, été 1971, p. 8-17.

«Poèmes ", Stratégie, 1, hiver 1972, p. 65-72.

«Nouveaux poèmes », Stratégie, 2, printemps-été 1972, p. 81-89.

"French mots savants ", les Herbes rouges, 6, septembre 1972, [n.p.].

«Inachevé va depuis de nombreuses années ", Cheval d'attaque (Paris), (numéro intitulé *Poésie au Québec, une année de lectures"), 16-17, juin 1976, p. 50-54.

«Poèmes et autres textes. - Cette poudrerie... ", Liberté, XX, 6, novembredécembre 1978, p. 51.

« Le monde sensible encore une fois ", Estuaire, 14, décembre 1979, p. 109-122.

«L'écriture commence par un rêve", la Nouvelle Barre du jour, 90-91, mai 1980, p. 11-32.

*Ainsi comme pour la première fois ", Liberté, XXII, 3, n 129, mai-juin 1980, p. 11-20.

«Inédits ", la Nouvelle Barre du jour, 92-93, juin 1980, p. 77-84.

*La création du monde ", la Nouvelle Barre du jour, 99, février 1981, p. 43-61.

"Entendez-vous ?", Estuaire, 24, été 1982, p. 61-77.

*(Brisure). Fragment », Liberté, XXV, 1, nº 145, février 1983, p. 30-31. 
*Visite à la mélancolie », Estuaire, 32-33, été-automne 1984, p. 37-38. Également paru dans Foldaan (Lavollon, France), 5, décembre 1984, p. 37-38.

"Linvention du réel ", Possibles, X, 1, automne 1985, p. 81-88.

"Trois poèmes extraits de "Là où nous sommes" ", le Sabord, automne 1990; 26, p. 36.

"Là où nous sommes », LitéRéalité, II, 2, automne 1990; p. 66-68. Également paru dans Estuaire, 57, septembre 1990, p. 15-20 et dans Trois, VI, 1, automne 1990, p. 69-72.

\section{I.3. Articles critiques ou essais parus dans des périodiques}

* La Nuit de la poésie: poésie politique ou poésie pure? *, le Devoir, 2 mai 1970, p. 14. [En collaboration avec Roger Des Roches.]

*Notes sur une pratique ", la Barre du jour, 29, été 1971, p. 2-7. [En collaboration avec Roger-Des Roches.]

"La matière du livre", préface à Roger Des Roches, l'Enfance d'yeux, Montréal, Éditions dụ Jour, 1972.

«Nicole devant son miroir en papier ", Presqu'Amérique, I, 8, août 1972, p. 3435.

«Denis Vanier. Violence, répercussions ", Presqu'Amérique, I, 10, octobrenovembre 1972, p. 22-24.

*Prolétaire un jour, prolétaire toujours», Presqu'Amérique, II, 1, janvier 1973, p. 23.

"Révolution, la littérature en critique ", Presqu'Amérique, II, 2, février 1973, p. 27-28.

"Littérature et lutte de classes (pratique textuelle/pratique formaliste)", Stratégie, 5-6, automne 1973, p. 111-121.

*Transgression et/ou littérature politique (esquisse)", la Barre du jour, 42, automne 1973, p. 33-43.

*Postface» à Roger Des Roches, Autour de Françoise Sagan indélibile. Poèmes et proses, 1969-1971, Montréal, l'Aurore, 1975, p. 89-98 (Lecture en vélocipède, $\mathrm{n}^{\circ} 15$ ).

"Comment ça s'écrit », Chroniques, I, 2, février 1975, p. 8-11. [Sur Philippe Haeck.]

"Quelle révolution?", Chroniques, 22, octobre 1976, p. 56-65.

"La lutte idéologique dans le champ culturel ", Chroniques, 23, novembre 1976, p. 38-65.

"Le corps de la peinture ", Chroniques, 23, novembre 1976, p. 13-27.

«Le corps de la peinture (partie 2)», Chroniques, 24-25, décembre 1976janvier 1977, p. 41-47.

«Une revue libre et légère et vide comme l'air *, Chroniques, 24-25, décembre 1976-janvier 1977, p. 114-118. [Sur Liberté.]

"Lettre ouverte au collectif ", Chroniques, 29-32, automne 1977-hiver 1978, p. 8-9. [En collaboration avec Marcel Saint-Pierre, André Morf et Jocelyne Lefebvre]. 
«Soulever une contradiction ", Chroniques, 29-32, automne 1977-hiver 1978, p. 317-318. [Sur André Roy.]

« Noir sur blanc. Bernard Labrousse. De l'idéologie dominée ", Dérives, 19, 1979 , p. 55-62.

«La poésie l'incroyable », Possibles, III, 2, hiver 1979, p. 109-111.

* Opinion. Pour Gauvreau », le Devoir, 19 mai 1979, p. 25.

"Enquête. Une question sans fin posée à la langue et au monde ", Liberté, XXIII, 2, no 134, mars-avril 1981, p. 115-118.

"L'imagination extérieure », la Nouvelle Barre du jour, 107, novembre 1981, p. 77-87.

"La poésie ce n'est jamais ça ", le Devoir, 28 novembre 1981, p. 30.

"Nicole Brossard, écrivain classique», la Nouvelle Barre du Jour, 118-119, novembre 1982, p. 71-75.

«Limpossible achèvement...», Possibles, VIII, 3, printemps 1984, p. 130-134.

"Irrévérence. À propos de la modernité de gauche et du retournement à soi ", dans En collaboration; Qui a peur de l'écrivain?, Montréal, les Herbes rouges, 1984, p. 3-25 ( $\left.\mathrm{n}^{\circ} 123-124\right)$.

"Une histoire injustifiable», la Nouvelle Barre du jour, 141, septembre 1984, p. 65-66.

*Le poème est le récit exact d'un instant de ce monde», Estuaire, 37, novembre 1985, p. 15-20.

"Témoignages d'écrivains", Espaces imaginaires d'ici et d'ailleurs. Textes et contextes $n^{\circ} .5$, deuxième partie, Laval, éd. Mondia, 1988, p. 341-342.

*Opinions *, Nuit blanche, 36, juin-août 1989, p. 46.

«Si j’aime et si j'écris de la poésie... ”, le Bonnet de nuit, I, 1, décembre 1990, p. 6.

\section{I.4. Mémoire}

«Langue(s)», mémoire de maîtrise ès arts (études littéraires). Montréal, Université du Québec à Montréal, 1979, $133 \mathrm{f}$.

\section{ENTREVUES}

GAUDET, Gérald, *Vivre de sa plume au Québec", Lettres québécoises, 47, automne 1987, p. 11-13.,

HAECK, Philippe et Patrick STRARAM, "Entretien. L'écriture change", Chroniques, I, 3, mars 1975, p. 8-27.

MARTIN, Jean-Guy, «François Charron ou la poésie au service des idées ", le Journal de Montréal, 3 octobre 1981, p. 40.

NEPVEU, Pierre, *Entrevue. François Charron. L'urgence de l'écriture", Lettres québécoises, 18 (été 1980), p. 40-47.

ROYER, Jean, «François Charron. "Toucher à la mère" ", le Devoir, 8 mars 1980 , p. 18. Reproduit dans Ecrivains contemporains. Entretiens 2, Montréal, l'Hexagone, 1983, p. 77-85. 
"Entretien avec François Charron", Foldaan (Lavollon, France), 5, 1984, p. 48-51.

\section{III. ÉTUDES}

\section{III.1 Études générales}

AHMED, Maroussia, «François Charron», The Oxford Companion to Canadian Literature, Toronto, Oxford et New York, Oxford University Press, 1983, p. 111-112.

[ANONYME], «Attribution du prix Émile-Nelligan à François Charron", Québec hebdo, I, 43, 10 décembre 1979, p. 4.

BAYARD, Caroline, The New Poetics in Canada and Quebec, From Concretism to Post-Modernism, Toronto, University of Toronto Press, 1989, 374 p. passim.

BEAUDET, André, *Éloge du passeur", Interventions du parlogue 1, les Herbes rouges, 1985 , p. $45-50\left(n^{\circ} 137\right)$.

BLAIN, Danièle, "Le prix Nelligan décerné au poète François Charron", le Journal de Montréal, 28 novembre 1979, p. 61.

CHAMBERLAND, Roger, «Circa les Herbes rouges. Modernité, post-modernité et avant-garde ", thèse de $\mathrm{Ph} . \mathrm{D}$., Québec, université Laval, 1988, $336 \mathrm{f}$.

CORRIVEAU, Hugues, «Des Herbes rouges ", la Nouvelle Barre du Jour, 70, octobre 1978, p. 51-78.

DESGENT, Jean-Marc, * 18 Assauts et autres recueils de poésies de François Charron ", Dictionnaire des ouvres littéraires du Québec, tome V, 19701975, Montréal, Fides, 1987, p. 247-251.

DOSTIE, Gaëtan, * En toutes lettres. François Charron ou la Poésie et le Politique ", le Jour, 29 juin 1974, p. V-3.

LECLAIR, Suzanne, «De l'importance de François Charron", le Devoir, 22 mars 1980, p. 24.

HAECK, Philippe, Naissances. De l'écriture québécoise, Montréal, VLB éditeur, 1979, 410 p. passim.

HAECK, Philippe, la Table d'écriture. Poéthique et modernité. Essais, Montréal, VLB éditeur, 1984, 386 p. passim.

HAMEL, Réginald, John HARE, Paul WYCZYNSKI, Dictionnaire des auteurs de langue française en Amérique du Nord, Montréal, Fides, 1989, p. 286-288.

MÉLANÇON, Robert, "Charron: l'impatience du poème", le Devoir, 8 décembre 1979, p. 23.

MILOT, Pierre; «L'avant-garde: processus institutionnel et conflits de légimitité ", l'Institution littéraire, sous la direction de Maurice Lemire avec la collaboration de Michel Lord, Québec, Institut québécois de recherche sur la culture, 1986, p. 117-127.

MILOT, Pierre, «Qui a peur de l'intellectuel en 1987?", Voix \& images, 36, printemps 1987, p. 530-534. [Reproduit avec variantes dans la Camera obscura du postmodernisme.] 
MILOT, Pierre, la Camera obscura du postmodernisme. Essais, Montréal, l'Hexagone, 1988, 90 p. (Essais littéraires).

MUIR, Michel, Poètes ou imposteurs?, Montréal, Louise Courteau, éditrice, 1985, 176 p. passim.

NEPVEU, Pierre, "L'ère de la sensation vraie *, dans l'Écologie du réel. Mort et naissance de la littérature québécoise contemporaine: Essais, Montréal, Boréal, 1988, p. 181-196, passim. (Papiers collés).

PELLETIER, Jacques, «Litinéraire de François Charron: des lendemains qui chantent au temps des incertitudes", l'Avant-garde culturelle et littéraire des années 1970 au Québec, sous la direction de Jacques Pelletier, Montréal, Université du Québec à Montréal, 1986, p. 99-118. (Cahiers du Département d'études littéraires, $n^{\circ} 5$ ).

PELLETIER, Jacques, «Constitution d'une avant-garde littéraire au Québec ", Etudes littéraires, XX, 1, printemps-été 1987, p. 111-130.

PELLETIER, Jacques, *L'art de la contestation dans les années 1970 au Québec ", Thèmes canadiens / Canadian Issues, VIII, 1987, p. 241-254.

ROYER, Jean, «Le prix Émile-Nelligan à François Charron, poète ", le Devoir, 29 novembre 1979, p. 21.

ROYER, Jean, «Qu'est-ce que la nouvelle écriture? », le Devoir, 8 mars 1980, p. 17.

ROYER, Jean, «L'anthologie (4). François Charron", le Devoir, 7 décembre 1985, p. 26.

ROYER, Jean, «François Charron», Magazine littéraire, 234, octobre 1986, p. 114-115.

THIBAUX, Hélène, «François Charron: insoutenable légèreté ou beauté cruelle de l'innocence? ", Estuaire, 55, hiver 1990, p. 72-75.

UNION DES ÉCRIVAINS QUÉBÉCOIS, Dictionnaire des écrivains québécois contemporains, recherche et rédaction Yves Légaré, Montréal Québec/Amérique, 1983, p. 95.

\section{III.2. Sur des recueils particuliers}

[ANONYME], «De quelques livres et quelques poètes", le Canada français, 21 février 1973, p. 56. [Sur Au "sujet » de la poésie.]

[ANONYME], «Poésie d'ici", la Presse, 17 février 1973, p. C-3. [Sur Au "sujet » de la poésie.]

BAYARD, Caroline, *Letters in Canada. 1981 [...] Poésie ", University of Toronto Quarterly, LI, 4, été 1982, p. 357-374. [Sur Mystère et 1980, p. 360-362.]

BAYARD, Caroline, «Dans la modernité, l'un change et l'autre tourne", Lettres québécoises, 28, hiver 1982-83, p. 39-42. [Sur Toute parole m'éblouira.]

BAYARD, Caroline, "Letters in Canada. 1982 [...] Poésie", University of Toronto Quarterly, LII, 4, été 1983, p. 358-370. [Sur Toute parole m'ébloui$r a$ et Littérature et nationalisme, p. 362-364.]

BAYARD, Caroline, *Letters in Canada. 1983 [...] Poésie», University of Toronto Qüarterly, LIII, 4, été 1984,.p. 360-372. [Sur Je suis ce que je suis, p. 369-370, et l'exposition *Crucifixions ", p. 360-361.] 
BAYARD, Caroline, «La démarche de François Charron ", Lettres québécoises, 47, printemps 1987, p. 48-49. [Sur le Fait de vivre et d'avoir vécu.]

BAYARD, Caroline, «La première et la troisième personnes ", Lettres québé-. coises, 55, automne 1989, p. 32. [Sur La beauté pourrit sans douleur.]

BEAUDET, André, «François Charron. Feu», Livres et auteurs québécois, 1978, p. 109-111.

BEAUDET, André, *Arts plastiques. Dialogue avec l'invisible ", Spirale, 34, mai 1983, p. 14 [Sur l'exposition * Crucifixions ".]

BEAULIEU, Ivanhoé, «François Charron: la recherche du nom ", la Presse, 26 décembre 1981, p. D-2. [Sur Feu et Mystère.]

BEAULIEU, Michel, *François Charron. Une cascade lyrique en 2500 lignes...!", Livres d'ici, 16, septembre 1981. [Sur 1980.]

BEAULIEU, Michel, «François ", Livre d'ici, X, 3, novembre 1984, p. 22.

BEAUSOLEIL; Claude, «François Charron », le Devoir, 7 novembre 1981, p. 27. [Sur. 1980.]

BEAUSOLEIL, Claude, «Des cris durables », le Devoir, 7 novembre 1981, p. 27. [Sur 1980.]

BEAUSOLEIL, Claude, «François Charron, le débordement des pages ", Les livres parlent, préface de Jean Royer, Trois-Rivières, Écrits des Forges, 1984, p. 74-75. [Sur 1980.]

BIRON, Michel, «L'œil du poète", Spirale, 105, avril 1991, p. 11. [Sur La beauté des visages ne pèse pas sur la terre.]

BLAIS, Jacques, * Letters in Canada. 1972 [...] Poésie ", University of Toronto Quarterly, XLII, 4, été 1973, p. 380-386. [Sur Au "sujet » de la poésie, p. 383.]

BLOUIN, Louise, * F. Charron. J.M. Desgent. Toute parole m'éblouira. Transfigurations ", Livres et auteurs québécois, 1982, p. 102-103.

BONIN, Éric, «La beauté pourrit sans douleur suivi de la Très Précieuse Qualité du vide", Nuit blanche, 37, septembre-octobre 1989, p. 15.

BOUVIER, Luc, «François Charron. Interventions politiques et Pirouette par hasard poésie », Livres et auteurs québécois, 1975, p. 112-114.

BROCHU, André, "En état de poésie ", Voix \& images, VIII, 1, automne 1982, p. 159-167. [Sur Mystère.]

BROCHU, André, «François Charron, etc. ", Voix \& images, IX, 2, hiver 1984, p. 145-154. [Sur Toute parole m'éblouira, D'où viennent les tableaux? et Je suis ce que je suis, p. 145-148.]

BROCHU, André, *De Gilles Hénault à King Kong *, Voix \& images, XI, 1, automne 1985, p. 125-134. [Sur François, p. 132-133.]

BROCHU, André, «Autour d'Alain Grandbois indélébile», Voix \& images, XI, 2, hiver 1986, p. 341-249. [Sur La vie n'a pas de sens, p. 346.]

BROCHU, André, «La fiction du réel/le réel de la fiction", Voix \& images, 35, hiver 1987, p. 322-330. [Sur le Fait de vivre ou d'avoir vécu, p. 323-324.]

BROCHU, André, "Quelques grands recueils", Voix \& images, 44, hiver 1990, p. 301-309. [Sur La beauté pourrit sans douleur, p. 301-302.] 
BRULOTTE, Gaétan, «L'injure », préface à Pirouette par hasard poésie, p. 7-9.

CHAMBERLAND, Roger, «La beauté pourrit sans douleur suivi de la Très Précieuse Qualité du vide. François Charron", Québec français, 75, automne 1989, p. 20.

CHAMBERLAND, Roger, "La Fragilité des choses. François Charron", Québec français, 70, mai 1988, p. 14.

CLOUTIER, Guy, *Territoire habitable», Nuit blanche, 27, mars-avril 1987, p. 16-17. [Sur La vie n'a pas de sens.]

COPPENS, Patrick, «Je suis ce que je suis ", Moebius, 20, hiver 1984, p. 71-72.

CORNELLIER, Louis, "La beauté psalmodiée", la Poésie au Québec. Revue critique 1989, Trois-Rivières et Joliette, Écrits des Forges et Cégep de Lanaudière, 1990, p. 41-42. [Sur La beauté pourrit sans douleur suivi de la Très Précieuse Qualité du vide.]

CORRIVEAU, Hugues, * La belle conduite ou le feu ", le Devoir, 10 septembre 1978, p. 18. [Sur Feu.]

CORRIVEAU, Hugues, «Poésie. L'étonnement devant Charron ", le Devoir, 10 mars 1979, p. 23. [Sur Blessures.]

CORRIVEAU, Hugues, «L'émotion de nommer ", Trois, V, 3, printemps 1990, p. 150-151. [Sur La beauté pourrit sans douleur suivi de la Très Précieuse Qualité du.vide.]

D'AlFONSO, Antonio, «D'où viennent les tableaux? Poèmes 1979", Nos livres, XV, 5688, avril 1984, p. 17.

D'ALFONSO, Antonio, "Charron (François). La vie n'a pas de sens", Nos livres, XVI, 6238, juin-juillet 1985, p. 27-28.

D'ALFONSO, Antonio; «Charron (François). Le Fait de vivre ou d'avoir vécu ", Nos livres, XVII, 6695, octobre 1986, p. 32-33.

DANSEREAU, Estelle, «En se défaisant", Canadian Literature, 106, automne, 1985, p. 106-108. [Sur François.]

DE BELLEFEUILLE, Normand, *Une bonne fin d'année pour les Herbes rouges ", la Presse, 26 février 1977, p. D-6. [Sur Enthousiasme.]

DE BELLEFEUILLE, Normand, «Petites et grandes misères de la critique ", Spirale, 21, septembre 1981, p. 4-5. [Sur 1980.]

DE BELLEFEUILLE, Normand, «Trafiquer le lisible», Spirale, 31, février 1983, p. 16. [Sur Toute parole m'éblouira.]

DEMERS, Jeanne, «François Charron. Littérature / Obscénités [...] Persister et se maintenir... ", Livres et auteurs québécois, 1974, p. 120-121.

DES ROCHES, Roger, "Prix Émile-Nelligan. Le doute royal», Spirale, 5, janvier 1980, p. 1. [Sur Blessures.]

DIONNE, René, * Letters in Canada. 1975 [...] Poésie », University of Toronto Quarterly, XLV, 4, été 1976, p. 346-352. [Sur Pirouette par hasard poésie, p. 351.]

DORION, Hélène, «Poésie via le Québec », Fond(s) de tiroir, Saint-Florentdes-Bois, décembre 1990, [n.p.]. [Sur La beauté des visages ne pèse pas sur la terre.] 
DUMONT, François, "Le Fait de vivre et d'avoir vécu. François Charron", Québec français, 64, décembre 1986, p. 12.

DUMONT, François, "Le Monde comme obstacle", Nuit blanche, 33, septembre-novembre 1988, p. 13.

FISETTE, Jean; «Poésie. Lorsqu'il est question de répertoire... Perrault, Charron, Des Roches, de Bellefeuille et les Franco-Américains ", Voix \& images, III, 2, décembre 1977, p. 325-328. [Sur Du commencement à la fin, p. 327.]

FISETTE, Jean, «François Charron. La Passion d'autonomie. Littérature et nationalisme », Livres et auteurs québécois, 1982, p. 265-267.

GAUDET, Gérald, *François Charron: "la précieuse leçon de la tendresse" ", le Devoir, 16 mai 1987, p. D-2. [Sur la Chambre des miracles.]

GAULIN, André, «Les Herbes rouges. Revue de poésie», Québec français, 34, mai 1979, p. 8-9. [Sur Blessures.]

GAULIN, André, «La Passion d'autonomie. Littérature et nationalisme. François Charron [...]", Québec français, 46, mai 1982, p. 11.

GIGUÈRE, Richard, "Letters in Canada. 1977 [...] Poésie ", University of. Toronto Quarterly, XLVII, 4, été 1978, p. 357-366. [Sur Du commencement à la fin et Propagande, p. 365-366.]

GIGUÈRE, Richard, «François Charron. Blessures [...]”, Livres et auteurs québécois, 1979, p. 104-106.

GIGUÈRE, Richard, "Letters in Canada. 1979 [...] Poésie ", University of Toronto Quarterly, XIIX, 4, été 1980, p. 359-368. [Sur Blessures, Peinture automatiste, le Temps échappé des yeux, p. 363-364.]

GIROUX, Robert, «François Charron. Du commencement à la fin", Livres et auteurs québécois, 1977, p. 137-138.

GIROUX, Robert, “ François Charron. Propagande», Livres et auteurs québécois, 1977, p. 138-139.

GONZAGUE, Louise de, "Charron (François). La Chambre des miracles", Nos livres, XVIII, 7011, mai 1987, p. 23-24.

GONZAGUE, Louise de, "Le Monde comme obstacle. Poésie de François Charron. Parfum, beauté, épines... ", Nos livres, XIX, 7, août 1988, p. 4-5.

HAECK, Philippe, “Au "sujet” de la poésie ", le Livre canadien, 145, 1973.

HAECK, Philippe, *La circulation des lettres. Les Herbes rouges polysèment ", Hobo / Québec, 14-15, janvier 1974, p. 23. [Sur Projet d'écriture pour l'été '76.]

HAECK, Philippe, «Interventions politiques", Chroniques, I, 3, mars 1975, p. 42-43.

HAECK, Philippe, «Persister et se maintenir dans les vertiges de la terre qui demeurent sans fin ", Chroniques, I, 3, mars 1975, p. 43-44.

HAECK, Philippe, «Être au service d'une cause: honorable mais difficile", le Devoir, 10 mai 1975, p. 24. [Sur Interventions politiques.]

HAECK, Philippe, "La poésie. Les mots et les masses ", le Devoir, 8 novembre 1975, p. 14. [Sur Pirouette par hasard poésie.] 
HAECK, Philippe, «Persister et se maintenir dans les vertiges de la terre qui demeurent sans fin ", le Livre canadien, VI, 333, novembre 1975.

HAECK, Philippe, «Pirouette par hasard poésie ”, Chroniques, I, 12, décembre 1975, p. 79.

HAECK, Philippe, «Du côté du matérialisme», Chroniques, 15, mars 1976, p. 49-52. [Sur Pirouette par hasard poésie.]

HAECK, Philippe, *François Charron. Peinture automatiste précédé de Qui parle dans la théorie? [...] ", Livres et auteurs québécois, 1980, p. 98-100.

HONS, Gaspard, *... de l'amour ", Espace de libertés (Belgique), jañvier 1991. [Sur La beauté des visages ne pèse pas sur la terre.]

LAFRANCE, Hélène, «François Charron. Feu, 1980, Mystère ", Livres et auteurs québécois, 1981, p. 147-150.

LEFEBVRE, Gordon, “La “Liberté des souffles” ", Spirale, 26, juin 1982, p. 3. [Sur la Passion d'autonomie.]

LEMIEUX, Jacques, «Des contradictions ", le Devoir, 30 mars 1974, p. 16 [Sur Littérature / Obscénités.]

LÉPINE, Stéphane, *Charrón (François). François ", Nos livres, XVI, 6116, mars 1985, p. 23.

LESSARD, Pierre, * L'amour du bout à l'autre du quotidien *, Bonnet de nuit, I, 1, décembre 1990, p. 26. [Sur La beauté des visages ne pèse pas sur la terre.]

MARQUIS, André, *Fantômes, dragons et cendres «, Lettres québécoises, 52 , hiver 1988-89, p. 40-42. [Sur la Fragilité des choses et le Monde.comme obstacle.]

MARQUIS, André, *Letters in Canada: 1989 [...] Poésie ", University of Toronto Quarterly, LX, 1, automne 1990, p. 50-58. [Sur le Monde comme obstacle et La beauté pourrit sans douleur.]

MARQUIS, André, *Prolongements ", Lettres québécoises, 61, printemps 1991, p. 32-34. [Sur La beauté des visages ne pèse pas sur la terre, p. 33.]

MARTIN, Raymond, «François Charron. François [...]”, Mobius, 23, 1984, p 87.

MAZEAUFROID, Jean, *François Charron. Peinture automatiste précédé de Qui parle dans la théorie?", Actuels (Frangy, France), 18, juillet 1981, p. 44-47.

MELANÇON, Benoît, «François ”, Spirale, 48, décembre 1984, p. 20.

MÉLANÇON, Robert, «François Charron. Un écrivain-peintre impatient ", le Devoir, 8 mars 1980, p. 19. [Sur Peinture automatiste, le Temps échappé des yeux et l'exposition * La couleur du noir ».]

MÉLANÇON, Robert, *François Charron bis », Liberté, XXV, 2, n 146, avril 1983, p. 76-78. [Sur la Passion d'autonomie et Toute parole m'éblouira.]

MICHON, Jacques, «Surréalisme et mobile», Etudes françaises, XI, 2, mai 1975, p. 124-125.

MORIN, Serge-J., *François Charron. Peinture automatiste", Vie des arts, XXV, 101, hiver 1980-1981, p. 86. 
NEPVEU, Pierre, «La jeune poésie. La critique peut-être... ", Lettres québécoises, 6 avril-mai 1977, p. 13-15. [Sur Enthousiasme.]

NEPVEU, Pierre, «Les Herbes rouges... jusqu'à François Charron*, Lettres québécoises, 11, septembre 1978, p. 38-40. [Sur Persister et se maintenir dans les vertiges de la terre qui demeurent sans fin et Du commencement ă la fin.]

NEPVEU, Pierre, «Feu la modernité?», Lettres québécoises, 23, automne 1981, p. 30-33. [Sur 1980, p. 32-33.]

NEPVEU, Pierre, «Haeck et Charron. Travailler à ne pas s'appartenir", Lettres québécoises, 25, printemps 1982, p. 36-39. [Sur Mystère.]

NEPVEU, Pierre, «Voyage dans le non-sens», Spirale, 54, septembre 1985, p. 9. [Sur La vie n'a pas de sens.]

NEPVEU, Pierre, “Nouveau lyrisme? „, Spirale, 65, novembre 1986, p. 6. [Sur le Fait de vivre et d'avoir vécu.]

OUELLET, Pierre, «Peinture automatiste précédé de Qui parle dans la théorie? et le Temps échappé des yeux, notes sur l'expérience de la peinture", Voix \& images, VI, 1, automne 1980, p. 161-163.

PAYANT, René, «La part des arts. François C. Lheureux prolifique ", Lettres québécoises, 18, été 1980, p. 48-51. [Sur Peinture automatiste et le Temps échappé des yeux.]

PIAZZA, François, "La voix au chapitre», Montréal-matin, 3 mars 1974, p. 30. [sur la Traversée / le Regard.]

PIAZZA, François, «Pirouette par hasard poésie», Montréal-matin, 2 novembre 1975, p. 10.

PIAZZA, François, *Poésie québécoise ", Montréal-matin, 2 novembre 1975, p. 18. [Sur Au «sujet » de la poésie.]

PILON, Jean-Guy, * Hébert. Saint-Germain. Laberge. Sabelia. Leblanc. Petit. Charron. Quelques questions sur le langage poétique", le Devoir (supp.), 19 mai 1973, p. xiv. [Sur $A u$ "sujet » de la poésie.]

POPOVIC, Pierre, «Abdiquer le poème ", Spirale, 90, septembre 1989, p. 14. [Sur La beauté pourrit sans douleur.]

RENAUD, Jacques, * La page des lecteurs. Propagande de François Charron, un ton dyonisiaque, une intuition héraclitéenne et un vieux manteau manichéen ", Lettres québécoises, 9, février 1978, p. 36.

RENAUD, Jacques, «La fraîcheur à gauche", le Devoir, 18 octobre 1977, p. 18. [Sur Enthousiasme, Du commencement à la fin et Propagande.]

ROBERT, Lucie, "À la recherche d'une poésie cruelle: les Herbes rouges", Livres et auteurs québécois, 1976, p: 117-119. [Sur Enthousiasme.]

ROYER, Jean, *Contre la douleur de l'effacement ", le Devoir, 15 novembre 1986, p. C-5. [Sur le Fait de vivre et d'avoir vécu.]

ROYER, Jean, «LAurore rajeunit le catalogue de la poésie québécoise ", le Soleil, 21 décembre 1974, p. D-7. [Sur Persister et se maintenir dans les vertiges de la terre qui demeurent sans fin.]

ROYER, Jean, «Quand l'écriture est un aveu ", le Devoir, 2 juillet 1983, p. 13.

[Sur Je suis ce que je suis.] 
SAINT-MARTIN, Fernande, * François Charron. Peinture automatiste. Pluralités », Livres et auteurs québécois, 1980, p. 272-275.

THÉBERGE, Jean-Yves, *De quelques livres et de quelques poètes", le Canada français, 21 février 1973, p. 56. [Sur Au « sujet » de la poésie.]

THÉBERGE, Jean-Yves, *Littérature / Obscénités, prétention et courage ", le Canada français, 10 avril 1974, p. 100.

THÉORET, France, «Dire l'époque», Spirale, 37, octobre 1983, p. 11. [Sur Je suis ce que je suis.]

THIBAUX, Hélène, «Poésies nues ou les profondeurs de la 'surface", Estuaire, 58, décembre 1990, p. 81-85. [Sur la beauté des visages ne pèse pas sur la terre.]

TOUPIN, Gilles, *Charron, le passeur ", la Presse, 31 décembre 1983, p. C-2. [Sur Je suis ce que je suis et D'où viennent les tableaux?]

TOUPIN, Gilles, *François Charron et l'éloge du vivant „, la Presse, 4 avril 1987, p. E-2. [Sur la Chambre des miracles.]

TOUPIN, Gilles, *La rédemption selon Charron ", la Presse, 13 janvier 1991, p. C-3. [Sur La beauté des visages ne pèse pas sur la terre.]

TOUPIN, Gilles; «Les incantations de François Charron ", la Presse, 20 mai 1989 , p. K-3. [Sur le Monde cómme obstacle et La beauté pourrit sans douleur.]

TRUDEL, Serge, * Charron (François). Je suis ce que je suis ", Nos livres, XIV, 5387, septembre 1983, p. 43-44.

VANIER, Denis et Josée YVON, «Projet d'écriture pour l'été '76", Hobo / Québec, 16-17, février 1974, p. 5.

VIAU, René, «Et voici pourquoi je peins...», le Devoir, 8 mars 1980, p. 26. [Sur l'exposition * La couleur du noir ".]

VIGNEAULT, Robert, "Le manifeste Refus global répercuté par François Charron. La passion d'autonomie. Littérature et nationalisme ", Lettres québécoises, 26, été 1982, p. 71-75.

YERGEAU, Robert, «Letters in Canada. 1985 [...] Poésie ", University of Toronto Quarterly, LVI, 1, automne 1986, p. 45-60. [Sur Blessures, p. 5253.]

YERGEAU, Robert, *Letters in Canada. 1986 [...] Poésie ", University of Toronto Quarterly, LVII, 1, automne 1987, p. 50-62. [Sur le Fait de vivre et d'avoir vécu, p. 58-59.] 